\title{
Propriedades psicométricas da versão portuguesa para jovens atletas de basquetebol do motivational climate scale for youth sports
}

\author{
Psychometric properties of the basketball portuguese version \\ of the motivational climate scale for youth sports
}

Carla Chicau Borrego e Carlos Silva

Research Center in Sports, Health Sciences and Human Development com o apoio da Federaçäo Portuguesa de Basquetebol

\section{Palavras chave: Clima Motivacional, Validação, Jovens, Atletas}

Resumo: O presente estudo analisa as propriedades psicométricas da versão portuguesa para jovens atletas de basquetebol do Motivational Climate Scale for Youth Sports (MCSYS). No estudo participaram 441 jogadores de basquetebol (206 masculinos e 235 femininos) com idades compreendidas entre 11 e 16 anos $(M=14.40$; SD=1.27). Como processo de validação, utilizou-se o método designado de abordagem por comité (committee approach), análise da consistência interna através do coeficiente de Alfa de Cronbach, análise fatorial exploratória (AFE) e análise fatorial confirmatória (AFC). A análise da confiabilidade do questionário foi considerada adequada, sendo os valores superiores a .70. Quanto aos indicadores de validade de constructo, obtidos por meio da AFC, apresentaram valores aceitáveis para todos os índices. Finalmente, encontrámos um apoio preliminar para a validade de constructo do MCSYS, evidência suficiente para avaliação do clima motivacional em jovens atletas de basquetebol.
Key Words: Motivational Climate, Validation, Young, Athletes The present study analyses the psychometric properties of the Portuguese athletes of basketball version of the Motivational Climate Scale for Youth Sports (MCSYS).). The sample comprises 441 baskeball athletes (206 males and 235 females), with a mean age of $14.40(\mathrm{SD}=1.27)$. The methods used in the MCSYS, validation process were: committee approach, internal consistency reliability via the Cronbach alpha coefficients, exploratory factor analysis and confirmatory factor analysis. According to literature, the analysis of the general reliability of the MCSYS and its dimensions generated adequate results, since all Cronbach alpha levels exceeded the value of 0.70. The factor validity indicatives, obtained through confirmatory factorial analysis, were acceptable for all indices. Lastly, we found preliminary support for the validity of the construct of the MCSYS, which is sufficient evidence to justify its use to measure the motivational climate in young Portuguese basketball athletes.

\section{Introdução}

Nas últimas duas décadas tem existido um aumento substancial na participação desportiva dos jovens. O estudo apresentado realça a participação desportiva dos jovens no contexto da modalidade de basquetebol. Os objetivos de realização e/ou critérios de sucesso têm sido identificados como uma importante e forte influencia na motivação desportiva. No entanto, alguns autores têm também valorizado a importância do contexto ou clima. O clima pode ser desenvolvido por treinadores, pais, pares e outros agentes significativos com influência no grupo. Duas formas de clima foram identificadas, refletindo o trabalho de Ames no contexto escolar . O clima para tarefa (mastery) é percecionado pelos colegas de equipa quando estes estáo diretamente direcionados para melhorar, ou aprender, o treinador e pais focalizam-se na aprendizagem e progresso individual, os erros são vistos como parte do processo, sendo o esforço. Por outro lado, um clima orientado para o Ego (Performance), é aquele em que existe comparação entre indivíduos, os erros são castigados e a desmonstraçáo de elevada capacidade é recompensada.

Ao nível da investigação, os instrumentos utilizados na análise dos objetivos de realizaçáo e do clima motivacional têm sido desenvolvidos recorrendo a populaçóes jovens/adultos. No entanto subsistem dúvidas sobre a capacidade de distinção plena entre as duas dimensóes pelos jovens com 10 a 15 anos de idade, os instrumentos utilizados para medir o clima motivacional, podem não ser adequados para este tipo de população. Para medir estes aspetos Ego e Tarefa em atletas jovens, os autores desenvolveram o Motivational Climate Scale for Youth Sports. Este instrumento, versão original, apresenta propriedades psicométricas adequadas, desenvolvidas com recurso a jovens atletas de várias modalidades, idades e género.

Considerando a importância de analisar o clima motivacional em jovens atletas, no presente estudo pretende-se descrever os processos de adaptação para jovens portugueses da modalidade de basquetebol, do MCSYS, e estudar as suas propriedades psicométricas. 


\section{Método}

\section{Participantes}

Participaram no estudo 441 jogadores de basquetebol, de ambos os sexos, com idades compreendidas entre 11 e 16 anos $(\mathrm{M}=14.40 ; \mathrm{SD}=1.27)$. Todos os atletas competem em campeonatos portugueses, pertencendo às seleçôes regionais de basquetebol. Para a validação do instrumento utilizamos 2 amostras independentes. Para a análise fatorial exploratória (AFE) e confirmatória (AFC) utilizamos amostra 1 (223 atletas com 11 a 14 anos, 98 masculino e 125 feminino; $M=13.34$, $\mathrm{SD}=.79$ ); e amostra 2 (218 atletas com 15 e 16 anos, 108 masculino e 110 feminino; $M=15.5, S D=.5)$. As amostras 1 e 2 foram utilizadas para a análise da consistência interna e validade de constructo do instrumento.

\section{Material e Procedimentos}

MCSYS, Motivational Climate Scale for Youth Sports, é um instrumento desenvolvido por Smith, et al. , para aceder ao clima motivacional em jovens atletas. A versão original é constituída por 12 itens, agrupados em 2 dimensóes: Ego (6 itens); Tarefa (6itens). Os atletas respondem numa escala de cinco pontos, 'Nada verdade (1) a 'Totalmente verdade (5).

TEOSQ foi utilizado para aceder à orientação para a tarefa e ego. É constituído por 13 itens (7-orientação Tarefa, e 6-orientação Ego), classificados numa escala de Likert de 5 pontos. A consistência interna do TEOSQ foi aceitável, sendo $\alpha=.76$ para a tarefa e $\alpha=.79$ para o Ego.

No processo de adaptação do MCSYS para Português utilizamos o método designado de abordagem por comité (committee approach) que consiste na avaliação do instrumento por parte de um grupo de pessoas bilingues que tenham conhecimentos aprofundados dos constructos teóricos medidos pelo questionário, compreendam as especificidades do contexto de aplicação e que estejam familiarizados comos princípios básicos do que é a avaliação psicológica . Primeiro recorremos a três profissionais bilingues (Português\&Inglês). A fase seguinte da tradução consistiu na análise das sugestóes por parte de três especialistas em Psicologia do Desporto e um treinador de basquetebol que dominassem o Inglês. Após a escolha da melhor opção para a tradução dos itens que compóem o questionário foi feito um estudo piloto com vinte atletas, com a mesma idade dos participantes do estudo, a fim de obter um feedback a cerca da linguagem utilizada e se esta se adequava aos atleta em questáo.

A fase seguinte consistiu na recolha de dados para a validação do instrumento. Após as consideraçóes éticas, autorização dos respetivos Coordenadores Técnicos, os atletas preencheram sem manifestarem dificuldades de compreensão o MCSYS, versão portuguesa. Para a análise e tratamento estatístico foram utilizados diversos procedimentos e análise disponíveis nos programas 'SPSS' e o 'AMOS ' for Windows (V20).

Numa primeira fase realizamos a análise descritiva dos dados (médias desvio padráo), e realizamos a AFE.

$\mathrm{Na} A F C$, para estimar os parâmetros utilizamos o método de Máxima Verossimilhança (ML). Uma vez que foi nosso objetivo medir a adequabilidade do instrumento ao modelo conceptual, na amostra do estudo, as medidas utilizadas para verificar a adequação do modelo aos dados foram: Quiquadrado $\left(\mathrm{c}^{2}\right)$ valor $\mathrm{p}$ náo significativo, rácio $\left[\left[\mathrm{c}^{2}\right]\right.$.inf.2] , Incremental Fit Index (IFI), Tucker-Lewis Index (TLI) e Comparative Fit Index (CFI), >.90, e o Root Mean Square Error of Approximation (RMSEA) <.06 .

Os coeficientes de alfa de Cronbach foram calculados para aceder à consistência interna dos 2 fatores, sendo o critério para aceitar valores $>$.70, Nunally .

\section{Resultados}

A análise descritiva dos dados de acordo com a estrutura proposta, revelou que os atletas recorreram às 5 possibilidades de resposta existentes para cada item. $\mathrm{Na}$ avaliação global das escalas obtivemos $\mathrm{M}=2.16 ; \mathrm{SD}=.65$ e $\mathrm{M}=4.35 ; \mathrm{SD}=.57$ para o Ego e Tarefa respetivamente. Os valores de assimetria e achatamento revelaram a não normalidade dos dados.

Para a EFA, o número de sujeitos utilizados no nosso estudo respeita o rácio de 10:1, , recorremos à solução com rotação oblíqua, que permite a correlação entre os fatores, uma vez que de acordo com a teoria subjacente ao modelo é expetável uma correlação negativa entre os fatores. Na tabela 1 verificamos que na soluçáo fatorial, resultante da rotaçáo obliqua, os pesos fatoriais para ambas as amostras se situam dentro dos valores considerados mínimos para serem interpretados, $>.30$. A AFE para ambas as amostra revelam que a solução de dois fatores explica aproximadamente $45 \%$ da variância total.

Tabela 1. -Análise Fatorial Exploratória

\begin{tabular}{lcccc}
\hline & \multicolumn{2}{c}{$11-14$ anos } & \multicolumn{2}{c}{$15-16$ anos } \\
\hline Factor & $\begin{array}{c}1 \\
\text { Ego }\end{array}$ & $\begin{array}{c}2 \\
\text { Tarefa }\end{array}$ & $\begin{array}{c}1 \\
\text { Ego }\end{array}$ & $\begin{array}{c}2 \\
\text { Tarefa }\end{array}$ \\
\hline Item1 & .40 & -.04 & .48 & .09 \\
Item3 & .59 & -.48 & .58 & -.45 \\
Item5 & .70 & .19 & .66 & .05 \\
Item8 & .75 & -.08 & .55 &.-27 \\
Item10 & .49 & -.12 & .58 & -01 \\
Item12 & .81 & .12 & .61 & .00 \\
Item2 & -.00 & .70 & .06 & .74 \\
Item4 & -.19 & .56 & .05 & .77 \\
Item6 & .07 & .74 & .08 & .80 \\
Item7 & -.07 & .56 & -.03 & .53
\end{tabular}




\begin{tabular}{|c|c|c|c|c|}
\hline & \multicolumn{2}{|c|}{ 11-14 anos } & \multicolumn{2}{|c|}{ 15-16 anos } \\
\hline Factor & $\begin{array}{c}1 \\
\text { Ego }\end{array}$ & $\begin{array}{c}2 \\
\text { Tarefa }\end{array}$ & $\begin{array}{c}1 \\
\text { Ego }\end{array}$ & $\begin{array}{c}2 \\
\text { Tarefa }\end{array}$ \\
\hline Item 9 & .09 & .81 & .02 & .79 \\
\hline Item 11 & -.04 & .73 & .00 & .61 \\
\hline
\end{tabular}

ML associado ao procedimento de bootstrapping, dado que o coeficiente de Mardia (normalidade multivariada) do modelo inicial foi elevado (67.25 modelo 1 e 68.05 modelo 2). Este procedimento permite assumir que os resultados das estimaçóes são robustos, não sendo desta forma afetados pela falta de normalidade multivariada.

CFA- Para estimar os parâmetros recorremos ao método de

Tabela 2. -Índices de Ajustamento do MCSYS

\begin{tabular}{lcccccccc}
\hline Modelo & Grupo & $\mathbf{n}$ & $\mathrm{c}^{2}$ & $\mathrm{c} 2 / \mathrm{df}$ & CFI & TLI & IFI & RMSEA \\
\hline 1 & $11-14$ anos & 223 & 135.56 & 2.55 & .87 & .83 & .87 & .08 \\
2 & $15-16$ anos & 218 & 100.83 & 1.90 & .92 & .90 & .92 & .06 \\
3 & Total & 441 & 135.56 & 2.55 & .87 & .83 & .87 & .08 \\
$4(\mathrm{a})$ & $11-14$ anos & 223 & 95.90 & 1,88 & .93 & .91 & .93 & .06 \\
$5(\mathrm{a})$ & $15-16$ anos & 218 & 92.08 & 1.80 & .93 & .91 & .93 & .06 \\
6 (a) & Total & 441 & 95.90 & 1.88 & .93 & .91 & .93 & .06 \\
\hline
\end{tabular}

NOTA: $c^{2}$ /df=Rácio Qui quadrado e graus de liberdade; CFI=Comparative Fit Index; RMSEA=Root Mean Square Error of Approximation; TLI=Tucker-Lewis Index; IFI=Bollen's Incremental Fit Index; (a)(cov 2 \&4; 9\&6)

Ao analisarmos a estrutura fatorial dos vários modelos, constituído por 2 fatores da versão portuguesa do MCSYS, relativamente ao ajustamento global do modelo, verificamos que o c2 é significativo para $\mathrm{p}<.001$, sugerindo um fraco ajuste, no entanto esta medida é muito sensível ao tamanho da amostra, devendo ser analisado outros indicadores. $\mathrm{O}$ valor do rácio c2 $/ \mathrm{df}<2$, à exceção dos modelos 1 e 3 .

Observa-se ainda que os valores dos índices de ajustamento comparativo ou incremental: TLI, CFI e IFI são $\geq .90$, , à exceção dos modelos 1 e 3. Também o índice da RMSEA expressou valores que se encontram de acordo com literatura . Nos modelos 1 e 3 realizamos modificaçóes (covariância entre o item $2 \& 4$ e 6\&9) que tiveram impacto nos modelos (CFI e IFI com .93). Os modelos 2, 4, 5 e 6 demonstraram bons índices, sugerindo que a escala se adequa ao modelo de dois fatores propostos.

Relativamente às soluçóes estandardizada encontradas, revelaram valores moderados, existindo valores de .40 . Sendo os valores dos erros (resíduos) observados moderados.

Para a análise da consistência interna utilizamos a associação entre a amostra $1 \& 2(n=441)$ constatando-se que os valores de alfa não poderiam ser elevados a partir da eliminação de itens. O cálculo do alfa de Cronbach para cada um dos fatores do modelo observado revelou que estes apresentaram uma consistência interna aceitável ( $\alpha=.70$ e $\alpha=.80$, respetivamente para o fator 'Ego e 'Tarefa').

Em todos os modelos analisados, foram identificadas correlaçôes negativas entre as escalas Tarefa e Ego, tal como preconizado no modelo original.

Validade concorrente MCSYS - Foram realizadas co- rrelaçóes simples entre a Orientação para a Tarefa e Ego (TEOSQ) e as escalas Tarega e Ego do MCSYC. Baseado em trabalhos anteriormente realizados onde examinaram a associação entre o clima motivacional e a orientação, foi postulado uma relação positiva entre a perceção de orientação para o Ego-Clima motivacional para o Ego, a perceção de orientação para o Tarefa-Clima motivacional para o Tarefa, e uma associação negativa entre o cruzamento entre as escalas (Ego e Tarefa) dos dois questionários. A escala Ego do MCSYS apresenta correlaçóes positivas .38 e negativas -.20 $(\mathrm{p} \leq .001)$ com a Orientação para o Ego e Tarefa, respetivamente. A escala Tarefa do MCSYS revelou correlaçóes negativas -.18 e positivas .50 ( $\mathrm{p} \leq .001)$ com a Orientaçáo para o Ego e Tarefa, $(\mathrm{p} \leq .001)$. Estes resultados são consistentes com as espectativas teóricas sobre a associação entre o clima motivacional e a orientação.

\section{Discussão}

A investigação realizada a jovens e crianças atletas tem sido desenvolvida na sua maioria com instrumentos desenvolvidos em população adultas e/ou jovens adultos. Nesse sentido e como salientam Smith, et al. é necessário o desenvolvimento e adaptaçáo de escalas para a população mais jovem, sendo esse o objetivo do estudo para a modalidade de basquetebol.

Relativamente à AFE verificamos que na solução encontrada existe uma correlação negativa entre os fatores tal como preconizado na modelo original de Smith, et al. . No que se refe aos pesos fatoriais registámos valores aceitáveis, no entanto o item 1 é aquele em que os valores são mais baixos. A 
AFC foi realizada recorrendo a duas amostras independentes com atletas de basquetebol com 11 a 14 anos (modelo 1e 3a), 15 a 16 anos (modelo 2 e 4a) e 11 a 16 anos correspondente à totalidade da amostra (3 e 6a). Como a versão original do instrumento foi validada para atletas de várias modalidades com idades entre os 9 e 14 anos, e considerando a facilidade de perceçáo dos atletas dos itens e constructos a este associados, assim como a dimensão do instrumento, e a necessidade de uma análise multidimensional (bateria de teste) da participação desportiva dos jovens atletas considerámos que este seria um instrumentos de eleição para analisar o clima motivacional em jovens atletas com 11 a 16 anos, reduzindo desta forma a resistência dos atletas às suas respostas. Os resultados da AFC em todos os modelos, confirmam a estrutura de dois fatores propostos, no entanto nos modelos 1 e 3, foi necessário introduzir melhorias (covariância entre itens), resultando nos modelos (4a e 5 a) com índices considerados como bons.

A versão portuguesa do MCSYS para atletas da modalidade de basquetebol com idades entre os 11 e 16 anos, apresenta níveis de consistência interna adequados $(\alpha \geq 70$, Nunally, , com valores superiores para o fator Tarefa, em consonância com o estudo de Smith, et al.

Com o objetivo de determinar a validade de constructo, utilizamos o TEOSQ, que mede um constructo similar. Os resultados evidenciaram correlaçôes significativas e no sentido esperado em conformidade com a literatura existente.

A versão portuguesa do MCSYS para atletas jovens da modalidade de basquetebol revelou propriedades psicométricas adequadas, sendo um instrumento fácil de aplicar e avaliar, contento instruçóes simples que permitem a sua aplicação em conjunto com outros instrumentos, possibilitando uma análise multidimensional dos jovens praticantes de basquetebol.

No entanto, como o estudo da validade de um instrumento é um processo contínuo de análise, sugerimos o desenvolvimento de investigaçôes e estudos adicionais na análise das qualidades métricas do instrumento em diferentes modalidades, considerando que as evidências psicométricas aqui apresentadas são apenas preliminares. Apontamos como sugestóes para futuras investigaçóes da validade, a sua aplicaçáo em diferentes contextos desportivos diferentes desportos e a relação com outras variáveis como a ansiedade e coesão.

\section{Referências}

Ames, C. (1992). Achievement goals and adaptive motivational patterns: The role of the environment. In G. C. Roberts (Ed.), Motivation in sport and exercise (pp. 2161-2176). Champaign, IL: Human Kinetics.

Brislin, R. (1980). Translation and content analysis for oral and written material. In H.Triandis \& J. Berry (Eds.), Handbook of Cross-Cultural Psychology (Vol. 2, pp. 389 - 444). Needham Heights: MA: Allyn and Bacon.

Fonseca, A. M., \& Brito, A. P. (2005). A questão da adaptação transcultural de instrumentos para a avaliaçáo psicológica em contextos desportivos nacionais - o caso do task and Ego Orientation in Sport Questionnaire (TEOSQ). Psychologica, 39, 95-118.

Geisinger, K. (2003). Testing and Assessment in Cross-Cultural Psychology. In J. Graham \& J. Naglieri (Eds.), Handbook of Psychology. Assessment Psychology (pp. 95-117). New Jersey: : John Wiley \& Sons, Inc.

Hu, L., \& Bentler, P. M. (1995). Evaluating model fit. In R. H. Hoyle (Ed.), Structural equation modeling: Concepts, issues, and applications (pp. 7699): Thousand Oaks, CA:Sage.

Hu, L., \& Bentler, P. M. (1999). Cutoff criteria fot fit indexes in covariance structure analysis: Conventional criteria versus new alternatives. Structural Equation Modeling, 6(1), 1-55.
Kahn, J. H. (2006). Factor Analysis in Counseling Psychology Research, Training, and Practice: Principles, Advances, and Applications. The Counseling Psychologist, 34, 684-718.

Moreno, J. A., Conte, L., Martínez, C., Alonso, N., González-Cutre, D., \& Cervelló, E. (2010). Propiedades psicométricas del Peer Motivational Climate in Youth Sport Questionnaire (PeerMCYSQ) con una muestra de deportistas españoles. Revista de Psicología del Deporte, 20(1), 101118.

Nunnally, J. C. (1978). Psychometric theory. New York: McGraw-Hill Book Company.

Smith, R. E., Cumming, S. P., \& Smoll, F. L. (2008). Development and Validation of the Motivational Climate Scale for Youth Sports. Journal of Applied Sport Psychology, 20(1), 116 - 136.

Ullman, J. B. (2001). Structural Equation Modeling. In B. G. Tabachnick \& L. S. Fidell (Eds.), Using Multivariate Statistics (4 ed., pp. 697-702). Needham Heights: MA: Allyn \& Bacon.

Worthington, R. L., \& Whittaker, T. A. (2006). Scale Development Research: A Content Analysis and Recommendations for Best Practices The Counseling Psychologist, 34(6), 806-838. 